University of Texas at El Paso

ScholarWorks@UTEP

$10-2019$

\title{
How to Reconcile Randomness with Physicists' Belief that Every Theory Is Approximate: Informal Knowledge Is Needed
}

\author{
Ricardo Alvarez \\ The University of Texas at El Paso, ralvarezlo@miners.utep.edu \\ Nick Sims \\ El Paso Community College, nsims2@my.epcc.edu \\ Christian Servin \\ El Paso Community College, cservin1@epcc.edu \\ Martine Ceberio \\ The University of Texas at El Paso, mceberio@utep.edu \\ Vladik Kreinovich \\ The University of Texas at El Paso, vladik@utep.edu
}

Follow this and additional works at: https://scholarworks.utep.edu/cs_techrep

Part of the Applied Mathematics Commons, and the Physics Commons

Comments:

Technical Report: UTEP-CS-19-102

\section{Recommended Citation}

Alvarez, Ricardo; Sims, Nick; Servin, Christian; Ceberio, Martine; and Kreinovich, Vladik, "How to Reconcile Randomness with Physicists' Belief that Every Theory Is Approximate: Informal Knowledge Is Needed" (2019). Departmental Technical Reports (CS). 1378.

https://scholarworks.utep.edu/cs_techrep/1378

This Article is brought to you for free and open access by the Computer Science at ScholarWorks@UTEP. It has been accepted for inclusion in Departmental Technical Reports (CS) by an authorized administrator of ScholarWorks@UTEP. For more information, please contact Iweber@utep.edu. 


\title{
How to Reconcile Randomness with Physicists' Belief that Every Theory Is Approximate: Informal Knowledge Is Needed
}

\author{
Ricardo Alvarez ${ }^{1}$, Nick Sims ${ }^{2}$, Christian Servin ${ }^{2}$, \\ Martine Ceberio ${ }^{1}$, and Vladik Kreinovich ${ }^{1}$ \\ ${ }^{1}$ Department of Computer Science \\ University of Texas at El Paso \\ $500 \mathrm{~W}$. University \\ El Paso, TX 79968, USA \\ ralvarezlo@miners.utep.edu,mceberio@utep.edu, \\ vladik@utep.edu \\ ${ }^{2}$ Computer Science and \\ Information Technology Systems Department \\ El Paso Community College (EPCC) \\ 919 Hunter Dr. \\ El Paso, TX 79915-1908, USA \\ nsims2@my.epcc.edu, cservin1@epcc.edu
}

\begin{abstract}
In this paper, we show that physicists' intuition about randomness is not fully consistent with their belief that every theory is only approximate. We also prove that there is no formal way to reconcile these two intuitions, this reconciliation has to be informal. Thus, there are fundamental reasons why informal knowledge is needed for describing the real world.
\end{abstract}

\section{Randomness: An Algorithmic Description (a brief reminder)}

From the computational viewpoint, what sequences of measurement results can we expect: case of a fixed physical theory. The usual analysis of algorithms and computability takes into account what we can compute "from scratch", by using only the given input and performing appropriate computational steps; see, e.g., [4].

In principle, in addition to the given input, we could also use the results of physical experiments. We normally do not use these results when performing un- 
related computations, since we do not expect the results of physical experiments to help us solve complex mathematical problems. It would be very strange to find, e.g., some radioactive material whose emissions will solve a given NP-hard problem like propositional satisfiability. We may get some help in solving this problem from other people, maybe from signals sent to us by an alien civilization - in short, from someone with intelligence - but we do not expect such help from an (unintelligent) physical process.

This intuitive idea has been formally described in [2] in terms of the important notion of Kolmogorov complexity (see, e.g., [3]). Let us briefly recall the corresponding formalization.

Kolmogorov complexity: a brief reminder. To understand this notion, we need to recall that while computers can process many different types of objects arrays, graphs, images, videos, etc. - in a computer, every object is represented as a sequence of $0 \mathrm{~s}$ and $1 \mathrm{~s}$, i.e., to use a computer science terminology, as a binary sequence.

The notion of Kolmogorov complexity was invented to formalize another intuitive idea - that of a random binary sequence. From the purely mathematical viewpoint, if we consequently flip a coin and write down all heads as $1 \mathrm{~s}$ and all tails as 0 s, then all length- $n$ sequences of 0 s and $1 \mathrm{~s}$ have the exact same probability $2^{-n}$. From this viewpoint, they are equally probable and thus, seem to have an equal right to be called random. In particular, for a large $n$, a sequence that we will actually get after flipping the coin $n$ times has the same probability as the sequence $0101 \ldots 01$, in which the sequence 01 is repeated many times. However, intuitively, the seemingly lawless sequence that we will actually get after flipping a coin looks random, while the sequence $0101 . .01$ does not look random - and it would be very surprising if such a sequence indeed appears as a result of flipping a coin. Moreover, if something like this sequence will be observed in a casino, after a while we will be absolutely sure that cheating is taking place (from: going on), and that the corresponding slot machine is not truly random (as it is supposed to be).

Kolmogorov noticed that there is a difference between sequences which are intuitively random and sequences which are not intuitively random: namely, the reason why we do not believe that the sequence $0101 \ldots 01$ is truly random is that it can be generated by a reasonably short program, in which we print 01 in a loop. On the other hand, if we consider a real sequence of 0 s and 1 s obtained by flipping a coin, we do not expect to find any regularity there, so the only way to generate this sequence is to print it bit-by-bit.

In other words, a sequence like $0101 . . .01$ can be generated by a very short program, while to generate the binary sequence $01 \ldots$ corresponding to the actual flipping results, we cannot use any program which is much shorter than a straightforward bit-by-bit printing program print $(01 \ldots)$ whose length is practically equal to the length of the binary string. To describe this difference in precise terms, Kolmogorov introduced the notion of Kolmogorov complexity $C(x)$ - the shortest length of a program (in some fixed programming language) that generates the binary string $x$. In these terms, if the Kolmogorov complexity 
$C(x)$ is much smaller than the length len $(x)$ of the sequence, then this sequence is not random. On the other hand, if $C(x) \approx \operatorname{len}(x)$ - or, to be more precise, if $C(x) \geq \operatorname{len}(x)-c$ for some small $C$, then we can say that the sequence $x$ is random.

From regular Kolmogorov complexity to prefix Kolmogorov complexity. The above definition of randomness can be made even more intuitive if we take into account that we can flip the coin as many times as we want, so the coin-flipping sequence can be extended to any length, the fact that we stopped at some specific point should not change our opinion on whether this sequence is random or not. From this viewpoint, if it is not so easy to generate the binary sequence that we have so far, but much easier to generate its extension, then the resulting sequence should still be marked as not random. Generating an extension can be easier than the sequence itself: e.g., to generate a sequence $001001 . . .001001$ is somewhat easier than its initial segment 001001...00100 when we need to take special care of the last two 0s. To take this into account, we need to replace the original Kolmogorov complexity $C(x)$ with prefix Kolmogorov complexity $K(x)$, which is defined as the shortest length of the program that generates either the binary sequence $x$ itself or a binary sequence starting with $x$; see, e.g., [3].

Conditional Kolmogorov complexity and the notion of information. We are interested in describing how the possibility to use an auxiliary sequence $y$ can potentially affect our computations. In principle, when we use the bits from the given sequence $y$, we do not need to compute them and thus, it may be possible to have a shorter program for computing $x$. An analogy is that if we already have a program for computing $\sin (x)$, then computing $\sin ^{2}(x)$ is fast: all you need to do is access the value of the sine and square it. In contrast, if we needed to compute $\sin ^{2}(x)$ "from scratch", by using only basic hardware-supported arithmetic operations, we would need to perform a dozen or so operations - which would necessitate a much longer program.

From this viewpoint, it is reasonable to consider program for which an access to the $i$-th bit from the sequence $y$ can be done by simply writing something like $y[i]$. The shortest length of such a using-calls-to- $y$ program for computing either $x$ or its extension is known as the conditional prefix Kolmogorov complexity and is usually denoted by $K(x \mid y)$.

In these terms, the fact that the use of $y$ should not make computing $x$ easier means that the complexity $K(x \mid y)$ should not be much smaller than the complexity of computing it without using $y$, i.e., that we should not have $K(x \mid y) \ll K(x)$. Of course, possibly using $y$ does not mean that we have to, so when we compute $K(x \mid y)$, we consider all possible programs for computing $x$-including programs that do not use $y$ at all. Thus, the length $K(x \mid y)$ of the shortest $y$-using program for computing $x$ must be either shorter or of the same length as the length $K(x)$ of the shortest program that computes $x$ without using $y: K(x \mid y) \leq K(x)$.

Thus, the difference $K(x)-K(x \mid y)$ must be non-negative and not too large. This difference is known as information in $y$ about $x$; it is denoted by $I(x: y)$. 
Similarly to the definition of randomness, the fact that using $y$ should not affect our ability to compute $x$ can be described as $I(x: y) \leq c$ for some small $c>0$.

Levin's formalization of the above intuitive idea: motivations. The above description is exactly what Levin used in formalizing the commonsense idea that the results of physical experiments should not help us solve mathematical problems.

All mathematics can be (and have been) formulated in terms of set theory ST, e.g., in terms of the usual Zermelo-Frenkel axiomatics ZF. In these terms, solving a mathematical problem - i.e., checking whether a given mathematical statement is true or not - is equivalent to checking whether a given statement from ST is true or not. Some mathematical problems are computational, not about proving results, but such problems can also be described in these terms: namely, if we want to compute the value of a real number (such as $\pi$ ), this means that for each $n$, we must find out whether the $n$-th digit in the binary expansion of this number is 0 or 1 - and the fact that the $n$-th digit is 1 is also a mathematical statement that can be, in principle, formalized in set theory.

Each statement from ST is a finite combination of symbols, so in a computer, it is represented as a sequence of $0 \mathrm{~s}$ and $1 \mathrm{~s}$ - which, in its turn, can be interpreted as a natural number. Moreover, it is easy to check whether a given natural number - i.e., in effect, a given sequence of $0 \mathrm{~s}$ and $1 \mathrm{~s}$ - is a syntactically correct statement of set theory. Thus, by trying all possible natural numbers $0,1,2$, $\ldots$, and checking whether each of them is a syntactically correct statement of set theory, we can enumerate all possible such statements into a sequence $S_{1}$, $S_{2}, \ldots$ In these terms, perfect mathematical knowledge can be represented by an infinite binary sequence $\alpha=\alpha_{1} \alpha_{2} \ldots$ where $\alpha_{i}=1$ if and only if the $i$-th statement $\alpha_{i}$ is true.

Similarly, all possible observation and measurement results can also be placed in a single binary sequence. Indeed, each observation and measurement result has to be represented in a computer - whether it is a numerical value or a textual observation - and can, thus, be naturally represented as a sequence of 0 s and 1s. Each measurement result can thus be described as a sequence of bits.

Descriptions of the corresponding experimental and observational settings - what is usually called metadata - can also be described in a computer. So, for each bit of each measurement result, we have a description, e.g., "the 5-th bit of measuring wind speed at UTEP campus at 12 pm on August 27, 2019". We can similarly sort such descriptions, and get a potentially infinite binary sequence $\omega=\omega_{1} \omega_{1} \ldots$ Of course, this sequence is only potentially infinite: at any given moment of time, we have only finitely many measurement and observation results, and thus, we only know a finite part of this potentially infinite sequence.

Levin's formalization of the above commonsense idea is that such that every $m$ and $n$, using the first $m$ bits of the measurement-results sequence $\omega_{1: m} \stackrel{\text { def }}{=}$ $\omega_{1} \ldots \omega_{m}$ does not help us compute the truth values $\alpha_{1: n} \stackrel{\text { def }}{=} \alpha_{1} \ldots \alpha_{n}$ of the first $n$ statements of set theory.

We already know how to formalize this "inability to help". Thus, we get the following precise description. 
Resulting Levin's formalization of the above intuitive idea. There exists a small integer $c>0$ for which, for all $m$ and $n$, we have $I\left(\alpha_{1: n}: \omega_{1: m}\right) \leq c$.

Comment. Levin called this formalization the Independence Postulate.

This formalization is in perfect accordance with modern physics. According to modern (quantum) physics, we have deterministic equations describing the wave function (i.e., the system's state). This state, in its turn, determines the probability of different measurement results, and the actual sequence of measurement results is random with respect to the corresponding probability measure; see, e.g., $[1,5]$.

For such sequence $\omega$ - which are random with respect to some computable probability measure - Levin's Independence Postulate is indeed true; see, e.g., [3]. In this sense, Levin's Independence Postulate is in perfect accordance with modern physics.

\section{Remaining Challenge and Our Proposed So- lution to This Challenge}

Physicists believe that every theory is approximate. It is good that modern quantum physics is in accordance with the physicists' intuition - which is formally described by Levin's Independence Principle.

But we should take into account that physicists have yet another intuition (see, e.g., [1, 5]): namely, many of them believe that no theory is final, that no matter what theory we formulate, no matter how well this theory describes the current experimental results, it will eventually turn out to be only a good approximation - there will be new experiments, new data that will require a modification of this theory.

This happened with Newton's mechanics - which needed to be modified to take into account relativistic and quantum effects, this will happen - many physicists believe - with modern relativistic quantum physics as well.

In other words, there will be no limit to progress of science: as science progresses, we will get more and more accurate models of reality.

How can we describe this belief in precise terms. In general, the above belief means that whatever physical law we come up with which is consistent with all physical experiments and observations so far, eventually we will come up with experimental data that violates this law. In terms of our notations, currently available results of experiments and observations simply form an initial fragment $\omega_{1: n}$ of the potentially infinite sequence $\omega$ of all such results. From the mathematical viewpoint, a physical law is simply a property $P\left(\omega_{1: n}\right)$ that limits possible values of such fragments to those that satisfy this property.

Thus, the above physicists' belief is that for each such property $P$, there exists an integer $M$ - corresponding to some future moment of time - at which the fragment $\omega_{1: M}$ will not satisfy the corresponding property $P$. 
Resulting challenge. In particular, the physicists' belief means that no matter what constant $c$ we select in our description of Levin's Independence Principle, there will be a value $M$ for which this principle will be violated, i.e., for which we will have $I\left(\alpha_{1: n}, \omega_{1: M}\right)>c$.

So, contrary to the physicists' intuition (and to modern physics), under this belief, a sequence of physical observations cannot be random - in the abovedescribed precise algorithmic sense of this randomness.

In other words, the two physicists' intuitions - the intuition about randomness and the intuition about infinite progress of physics - are not fully compatible. How can we reconcile these two intuitions?

How to reconcile the two intuitions: suggestion and challenges. Due to the second (progress-of-science) intuition, we cannot require - as Levine did - that all the values of the information $I\left(\alpha_{1: n}, \omega_{1: m}\right)$ are bounded by a constant. However, intuitively, the first (randomness) intuition tell us that we cannot expect too much information above complex statements by simply looking at nature. We do not expect that we can find a solution to a complex mathematical problem by simply measuring, the sizes of the tree leaves.

In other words, since we cannot require that the amount of information $I\left(\alpha_{1: n}, \omega_{1: m}\right)$ should be small - i.e., it should not grow too fast with $m$.

This idea is informal, can we formalize it? Unfortunately, not really: if we select some slowly growing function $c(m)$ and require that

$$
I\left(\alpha_{1: n}, \omega_{1: m}\right) \leq c(m),
$$

we will have the same problem as with the original Levin's Independence Postulate: that, according to the progress-of-science intuition, there will be some $M$ for which this inequality will be violated.

Thus, the only way to reconcile the two intuitions is to acknowledge that the only way to do that is to make an informal statement.

Thus, there are fundamental reasons why informal knowledge is needed for describing the real world.

\section{Acknowledgments}

This work was supported in part by the National Science Foundation grants 1623190 (A Model of Change for Preparing a New Generation for Professional Practice in Computer Science) and HRD-1242122 (Cyber-ShARE Center of Excellence).

\section{References}

[1] R. Feynman, R. Leighton, and M. Sands, The Feynman Lectures on Physics, Addison Wesley, Boston, Massachusetts, 2005. 
[2] L. A. Levin, "Randomness conservation inequalities: information and independence in mathematical theories", Information and Control, 1984, Vol. 61, pp. $15-37$.

[3] M. Li and P. Vitanyi, An Introduction to Kolmogorov Complexity and Its Applications, Springer, New York, 2008.

[4] C. Papadimitriou, Computational Complexity, Addison-Wesley, Reading, Massachusetts, 1994.

[5] K. S. Thorne and R. D. Blandford, Modern Classical Physics: Optics, Fluids, Plasmas, Elasticity, Relativity, and Statistical Physics, Princeton University Press, Princeton, New Jersey, 2017. 